\title{
INTEGRAL IMAGING THREE-DIMENSIONAL (3D) DISPLAY WITH WIDE-VIEWING ANGLE USING MULTIPLE ILLUMINATIONS
}

\author{
Md. Shariful Islam, Md. Tariquzzaman and Md. Zahidul Islam \\ Department of Information and Communication Engineering, Islamic University, \\ Kushtia-7003, Bangladesh
}

\begin{abstract}
In this paper, a three-dimensional (3-D) integral imaging (II) system to improve the viewing angle by using the multiple illuminations is proposed. In this system, three collimated illuminations that are directed to three different angles in order to get widen propagation angle of point light source (PLS). Among three illuminations two slanted illuminations increase the propagation angle of PLS over the conventional method. Simulation result shows that the viewing angle of proposed PLS displays is three times larger than conventional PLS displays. In the simulation, we used Light Tools 6.3 to reconstruct an object.
\end{abstract}

\section{KEYWORDS}

Integral Imaging 3D display, Multiple Illuminations, Point Light Source PLS

\section{INTRODUCTION}

Integral imaging which was first proposed by G. Lippman in 1908 [1], has been regarded as one of the most attractive 3D imaging and display techniques, because it can provide full-color, fullparallax and continuous-viewing images. In addition, integral imaging does not require viewingaids, multiple observers, and multi viewable. Integral imaging technique consists of two processes, pickup and reconstruction [2-6], as shown in Figure 1. In the pickup process of integral imaging, direction and intensity information of the rays coming from a 3D object are spatially sampled by use of a lens array (or pinhole array) and 2D image sensor. The lens array composed of many convex elemental lenses is positioned immediately in front of the photographic film as a capture device.

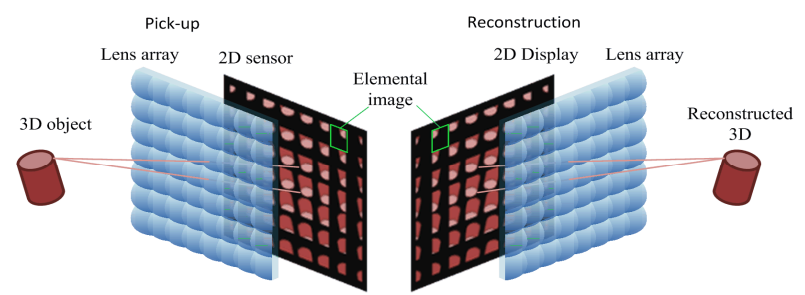

(a)

(b)

Figure 1. Basic concept of integral imaging. (a) Pick-up and (b) Reconstruction 
International Journal on Cybernetics \& Informatics (IJCI) Vol. 3, No. 4, August 2014

The captured image is composed of numerous small elemental images, which are imaged by lens array, with their number corresponding to that of the elemental lenses. Therefore, a size of elemental image is equal to a size of elemental lens. A real image, which is pseudoscopic (depthreversed) image [7-10], is reconstructed through the lens array when the elemental image is displayed on a 2D display device in Figure.1 (b). Although we are living in a three-dimensional (3D) world, almost all displays present two-dimensional (2D) images. 3D displays are needed for some important applications such as a virtual surgery, a 3D design, and a remote control, because 3D image includes extra information that is helpful to human. Therefore, 3D display is interesting research field. Some 3D technologies have been developed, for example, stereoscopy, autostereoscopy, holography and integral imaging. Among them, the integral imaging technology has some advantages that it does not require any special glasses and has continuous viewpoints within the viewing angle. It also provides full parallax, full color, and real time. However, conventional integral imaging display (IID) has drawbacks such as small viewing angle (VA), limited viewing resolution and short depth range [11]. In this paper, we focus on the VA issue.

\section{VIEWING ANGLE OF PLS DISPLAY}

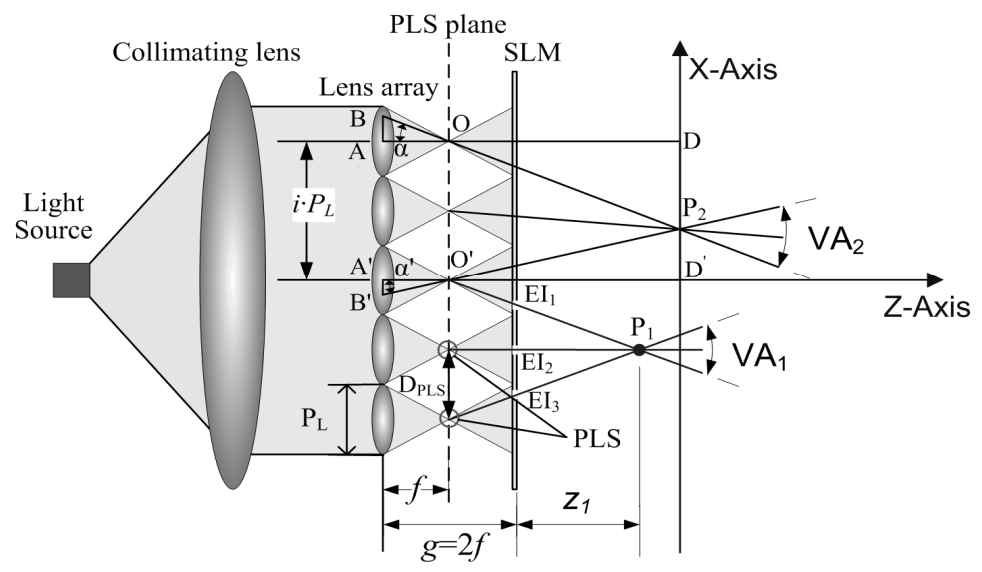

Figure 2. Conventional PLS display

Figure 2. shows a structure of PLS display that consists of a light source, a collimating lens, a lens array and a spatial light modulator (SLM). Collimated lights are focused by the lens array in a focal plane of the lens array. This focal plane is called PLS plane. A number of PLSs is equal to a number of elemental lenses, because conventional PLS display uses one plane illumination. When the SLM displays the elemental images, the lights from the PLS are modulated to create integrated 3D image. For example, three rays reconstruct 3D integrated point $\mathrm{P} 1$ at a cross section of those three rays when three pixels of SLM such as EI1, EI2, and EI3 open and other pixels close, as shown in Figure 2. The size of elemental image depends on a position of SLM. If a gap $g$ between SLM and the lens array is longer than the $2 \mathrm{f}$, the light beams from PLSs overlap in the SLM. When the $\mathrm{g}$ is shorter than the $2 \mathrm{f}$, PLSs does not illuminate some parts of SLM so those parts are not used. Therefore, the $g$ is equal to twice of focal length of the lens array. Each elemental lens creates one PLS in the focal plane hence a distance between two PLSs is equal to a size of the elemental lens PL. The distance between two PLSs is important parameter because it determines a spatial resolution of PLS display. Although the size of the elemental lens is small, the spatial resolution of PLS display increases, but it reduces an angular resolution due to reduced number of SLM pixels corresponding to each elemental lens. From Figure 2, 3D integrated point 
International Journal on Cybernetics \& Informatics (IJCI) Vol. 3, No. 4, August 2014

appears in the cross section of diverged rays from the PLSs so a viewing angle of PLS display is determined by diverging angle of the PLS [12]. That is given by $V A=2 \cdot \arctan \left(\frac{P_{L}}{2 f}\right)$.

where $P_{L}$ and $f$ are the size and the focal length of elemental lens. However, the viewing angles of each integrated points are different because the viewing angle depends on the position of 3D point. For example, viewing angles of $\mathrm{P}_{1}$ and $\mathrm{P}_{2}$ are different that are shown in Figure 2. Since $\alpha$ and $\alpha^{\prime}$ are angles of two extreme rays to reconstruct $\mathrm{P}_{2}$, the viewing angle of $\mathrm{P} 2$ is equal to

$$
V A=\alpha+\alpha^{\prime}=\arctan \left(\frac{A B}{f}\right)+\arctan \left(\frac{A^{\prime} B^{\prime}}{f}\right)
$$

From triangles $\mathrm{ABO}$ and $\mathrm{P}_{2} \mathrm{DO}$, we can write $\frac{A B}{P_{2} D}=\frac{A O}{D O}=\frac{f}{z_{2}}$,

where $z_{2}$ is a distance of $\mathrm{P}_{2}$ from the lens array. Since $\mathrm{P} 2 \mathrm{D}=i \cdot P_{L^{-}}$, the disparity is given by

$$
A B=\left(i \cdot P_{L}-x\right) \cdot \frac{f}{z_{2}+f}
$$

where $x$ is coordinate of $\mathrm{P}_{2}$ and $i$ is index of elemental lens that directs upper extreme ray of $\mathrm{P}_{2}$ in Figure 2. The disparity $A B$ has to be smaller $P_{L} / 2$ and greater $-P_{L} / 2$, because the size of elemental image is equal to elemental lens. We obtain the viewing angle of the upper extreme ray

$$
\alpha=\arctan \left(\frac{i \cdot P_{L}-x}{z_{2}+f}\right)
$$

By repeating previous steps, the angle of the down extreme ray is given

$$
\alpha^{\prime}=\arctan \left(\frac{x-i^{\prime} \cdot P_{L}}{z_{2}-f}\right)
$$

where $i^{\prime}$ is index of elemental lens that directs down extreme ray of $\mathrm{P}_{2}$. Hence, the viewing angle of integrated point at any position is defined

$$
V A=\alpha+\alpha^{\prime}=\arctan \left(\frac{i \cdot P_{L}-x}{z+f}\right)+\arctan \left(\frac{x-i^{\prime} \cdot P_{L}}{z+f}\right)
$$

The $i$ and $i^{\prime}$ are defined with indexes of elemental lenses that direct two rays with largest two disparities among collected ray to reconstruct 3D point. The largest disparity is half of the elemental lens. 
International Journal on Cybernetics \& Informatics (IJCI) Vol. 3, No. 4, August 2014
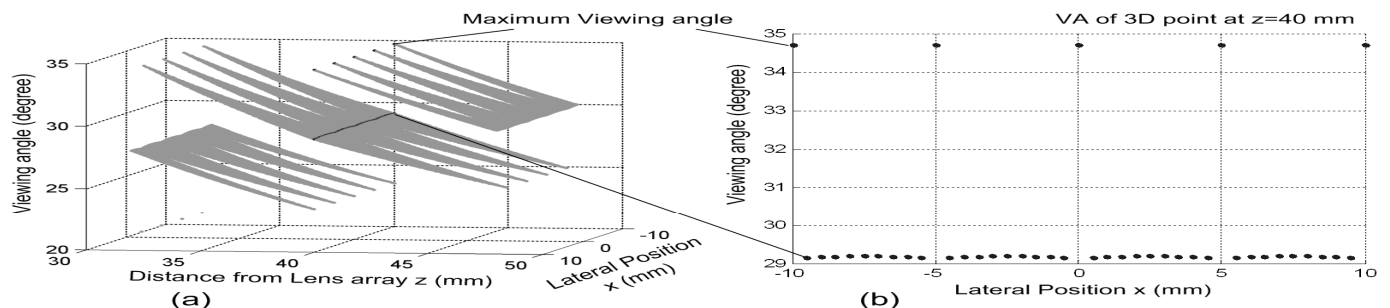

(a)

(b)

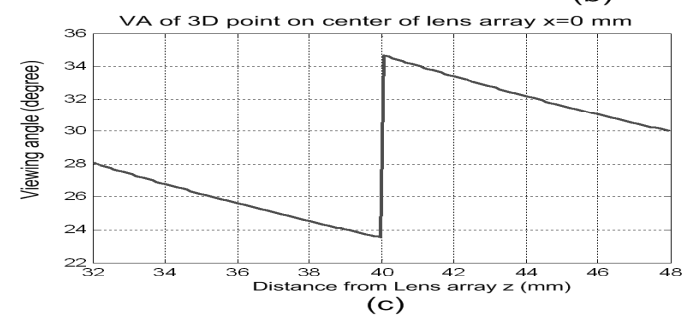

Figure 3. Calculation of the viewing angle of integrated points. (a) Dependent on the distance and the lateral position of integrated point. The viewing angles of integrated points where are (b) on palne at $z=40$ $\mathrm{mm}$ and (c) on the center of lens array

Figure 3. shows calculation of the viewing angle of integrated point that is determined an angle of between two extreme rays. In this calculation, a size, a focal length of the elemental lens, and a distance between the lens array and the display are $5 \mathrm{~mm}, 8 \mathrm{~mm}$, and $16 \mathrm{~mm}$, respectively. The distance of integrated point is from $30 \mathrm{~mm}$ to $50 \mathrm{~mm}$. The lateral position is from the center of lens array. According to Figure 3, the viewing angle of PLS display is larger than IID as the same configuration. The viewing angles depend on the collected ray number highly. When the disparity of elemental image is equal to half of the elemental lens, the viewing angle of that point achieves the maximum value. For example, viewing angles of pixels at $40 \mathrm{~mm}$ from SLM and lateral positions such as $-10 \mathrm{~mm},-5 \mathrm{~mm}, 0 \mathrm{~mm}, 5 \mathrm{~mm}$, and $10 \mathrm{~mm}$ are $34.76^{\circ}$ in Figure 3 . Those lateral positions are centers of elemental lenses ordered $-2,-1,0,1$, and 2. J.-H. Park et al. used the double collimated to increase the horizontal viewing angle of $3 \mathrm{D} / 2 \mathrm{D}$ convertible IID [13]. However, their method can enhance only horizontal viewing angle and requires different elemental images for each of double collimated illuminations.

\section{PRINCIPLE OF WIDE-VIEWING ANGLE PLS DISPLAY}

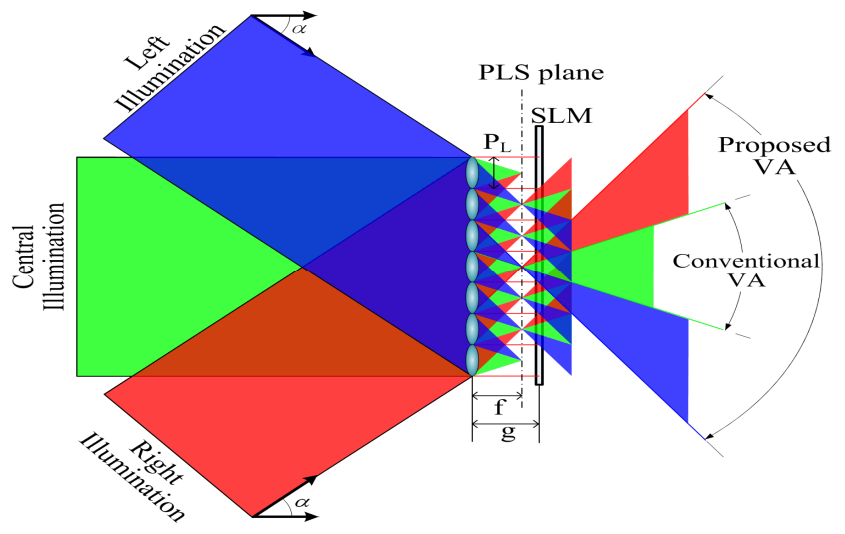

Figure 4. Structure of the wide-viewing angle display 
International Journal on Cybernetics \& Informatics (IJCI) Vol. 3, No. 4, August 2014

Figure 4. shows a structure of the proposed method. A conventional PLS display uses only center illumination but in our proposed method left, center, and right illuminated lights are collected by the lens array in the focal plane of the lens array and propagated into different directions. One elemental lens collects three different illuminations at the three different positions in the focal plane. Hence, one PLS consists of three parts of left, center, and right illuminations that are collected in focal point of elemental lens by three neighboring elemental lenses. The two additional parts of one PLS enhance the propagation angle of PLS so that total viewing angle of the proposed method is enhanced. The three parts of one PLS must be focused on one point where is the center of the corresponding central elemental lens in focal plane. Therefore, the left and right illumination are collected far $P_{L}$ from the center of corresponding lens. In this condition, an angle of illumination is given

$$
\alpha=2 \cdot \arctan \left(\frac{P_{L}}{f}\right) .
$$

Since the angle of diverging rays determine the viewing angle, the viewing angle of proposed method is given by

$$
V A=2 \cdot \arctan \left(\frac{3 P_{L}}{2 f}\right)
$$

From Equations (1) and (9), the viewing angle of proposed method is three times higher than the conventional PLS display, but both equations just infer the maximum viewing angle. The viewing angle of any point is defined by Eq. (6) but the indexes of two elemental lenses are calculated with different disparity. In the new method, the disparity is greater than $-3 \cdot P_{L} / 2$ and smaller than $3 \cdot P_{L} / 2$ because of additional two illuminations

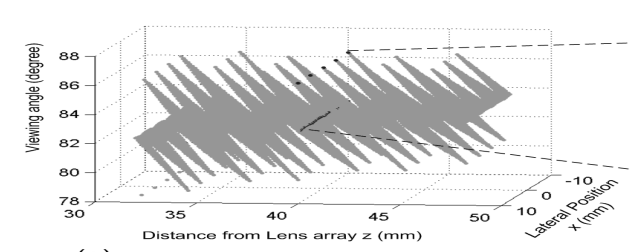

(a)

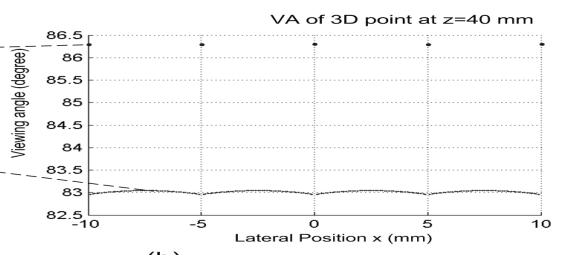

(b)

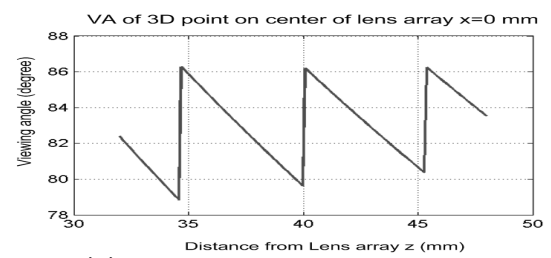

(c)

Figure 5. Viewing angle of proposed method depends (a) on the distance and the lateral position of integrated point. Viewing angles of integrated points where are (b) on plane at $40 \mathrm{~mm}$ from the SLM and (c) on the center of lens array

Figure 5. shows viewing angle calculation of proposed in the same image volumes as conventional PLS display. According to Figures. 3 and 5 the viewing angle of proposed method enhances with multiple plane illuminations. In Figure 5.(b) viewing angles of points in the plane at $z=40 \mathrm{~mm}$ is 2.86 times higher than conventional PLS display in the same configuration. In additional, the difference between maximum viewing angle and minimum viewing angle of proposed method is smaller than the conventional PLS display. From Figure 5.(c), this difference reduces when the distance of 3D point increases. The gap $g$ between SLM and lens array is 
International Journal on Cybernetics \& Informatics (IJCI) Vol. 3, No. 4, August 2014

important parameter, because the overlapping of elemental images and miss using parts of SLM depend on the gap. In those reasons, we can determine the gap

$$
g=\frac{3 f}{2}
$$

When $g$ is given Eq.10, the proposed method can display 3D image without overlapping problem and all parts of SLM are used.

\section{SIMULATION RESULS}

In the simulation, we used Light Tools 6.3 to reconstruct an object. A simulation setup is shown in Figure 6. Three plane light sources illuminate the lens array that the focal length $f$ is $3.3 \mathrm{~mm}$ and the size of elemental lens $P_{L}$ is $1 \mathrm{~mm}$. It is widely used lens array IID. A receiver of Light Tools is similar 2D sensor so we use an imaging lens to capture reconstructed object in the different viewer's positions.

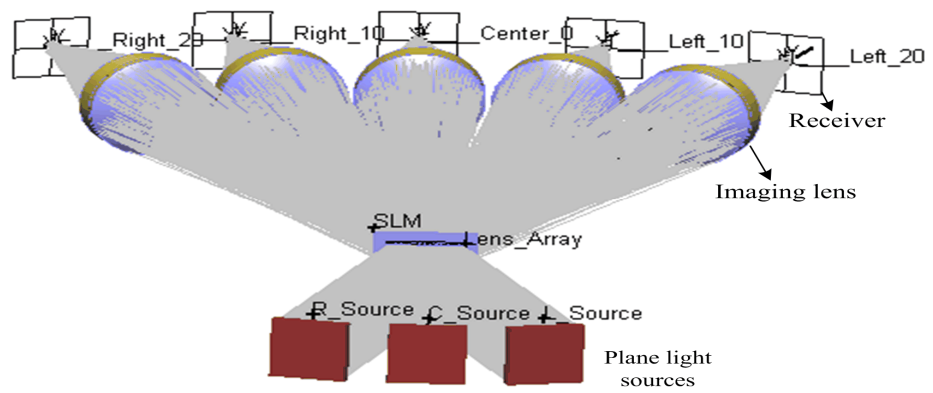

Figure 6. Implementation of wide-viewing angle PLS display with Light Tools

Figure 7. Show simulation result of ray collection. In Figure 7(a), three differently directed rays are collected by lens array in the three different positions because of Petzval curvature. It increases a size of PLS, as shown in Figure.7 (b). We use just three-plane illuminations arranged along horizontal axis. Theretofore, a width of PLS is bigger than height of PLS, as shown in Figure.7(b). If the lens array consists of perfect elemental lens, all rays are collected at PLS plane and the width and the height of PLS are same. The receiver is located at PLS plane, so we can determine a distance between two PLSs. It is equal to $1 \mathrm{~mm}$ from Figure.7 (b), since the size of the elemental lens is $1 \mathrm{~mm}$.

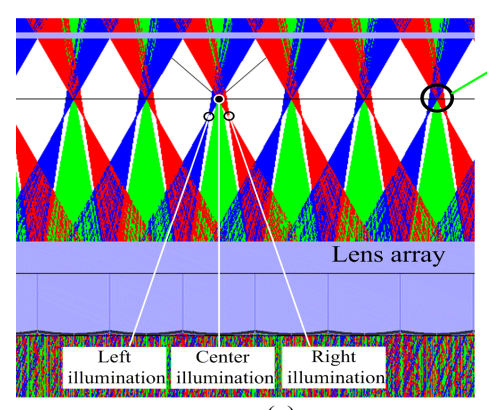

(a)

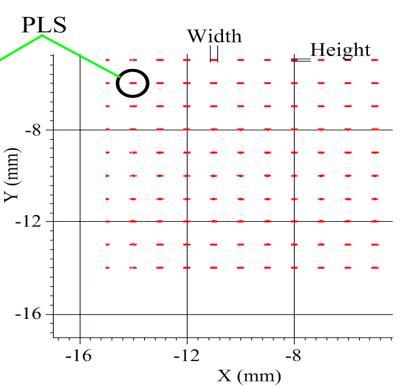

(b)

Figure 7. Ray collection (a) in top side and (b) in PLS plane 
International Journal on Cybernetics \& Informatics (IJCI) Vol. 3, No. 4, August 2014

Figure. 8 shows the reconstructed object with the conventional PLS and the proposed method at the five different positions such as a left $20^{\circ}$, a left $10^{\circ}$, a center $0^{\circ}$, a right $10^{\circ}$, and a right $20^{\circ}$. Since the imaging lens and the receiver are the same with single lens camera, the reconstructed object " 3 " is inverted. We do not see any object in Figures.8(a) and 8(e) because the left $20^{\circ}$ and the right $20^{\circ}$ are outside of the viewing angle of the conventional PLS display. In the proposed method, those two positions are inside of viewing angle of proposed method so the object is reconstructed in Figures.8(f) and.8(j). A quality of the reconstructed object depends on the number of rays.

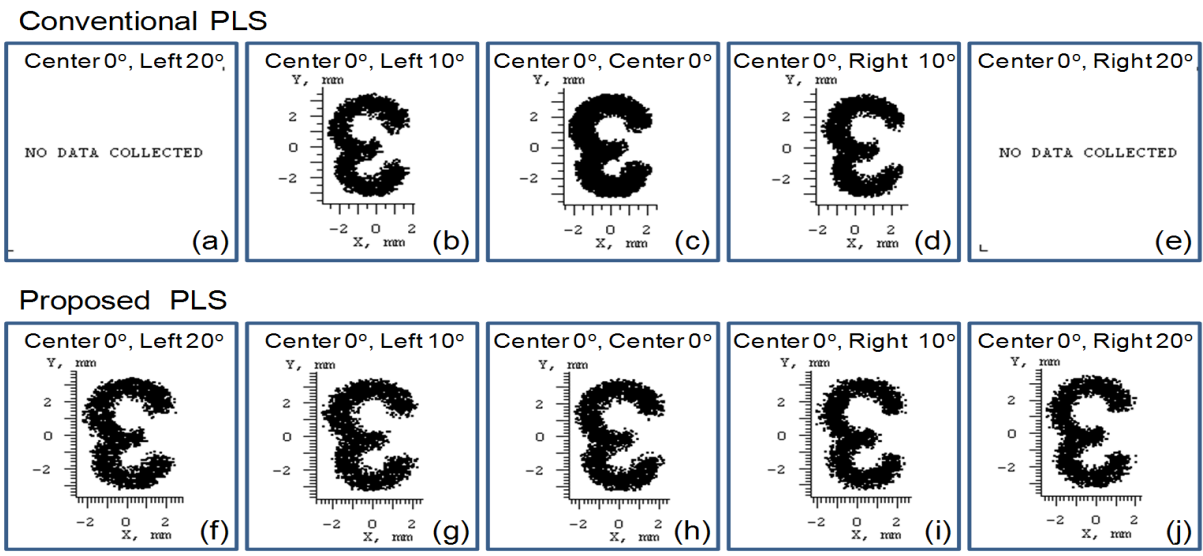

Figure 8. Reconstructions of (a)-(e) conventional PLS display and (f)-(j) proposed PLS display

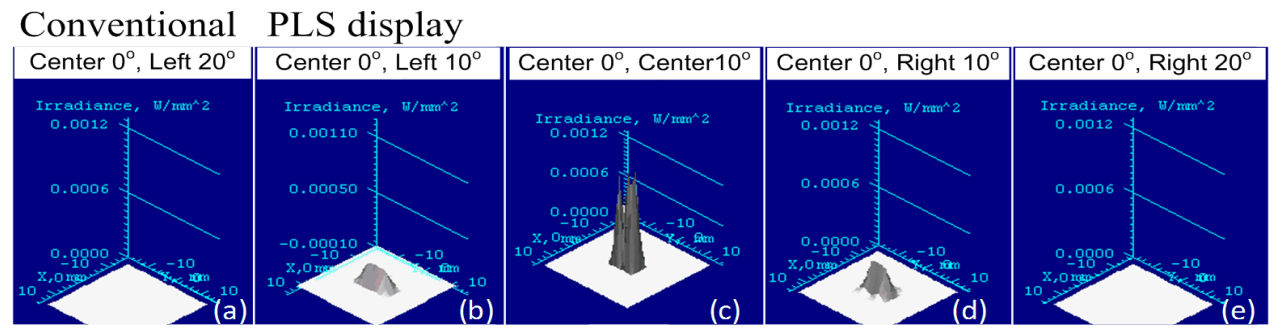

Proposed PLS display

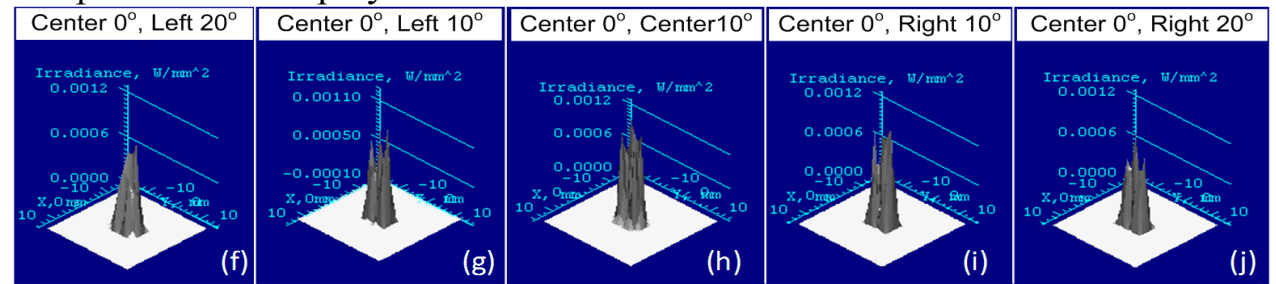

Figure.9 Intensity distributions of (a)-(e) conventional and (f)-(j) proposed PLS display at the five different viewing directions.

Figure 9. shows an intensity distributions of conventional and proposed PLS display at the five different positions such as the left $20^{\circ}$, the left $10^{\circ}$, the center $0^{\circ}$, the right $10^{\circ}$, and the right $20^{\circ}$. In the conventional PLS display, the intensity decreases if the angle of observer increases. When the angles of observer are as the left $20^{\circ}$ and the right $20^{\circ}$, observer does not see any object. From Figures.9 (f) - (j), the intensities of the object " 3 " are almost same in the five different viewing directions 
International Journal on Cybernetics \& Informatics (IJCI) Vol. 3, No. 4, August 2014

\section{CONCLUSION}

In conclusion, a method to enhance the viewing angle in II has been proposed and demonstrated by simulation. In this method, three collimated illuminations that are directed to three different angles in order to get widen propagation angle of point light source (PLS). Among three illuminations two slanted illuminations increase the propagation angle of PLS over the conventional method. Simulation result shows that the viewing angle of proposed PLS displays is three times larger than conventional PLS displays.

\section{ACKNOWLEDGEMENT}

This research work is supported by MICT (Ministry of Information and Communication Technology Division), Government of the People's Republic of Bangladesh [56.00.0000.028.33.007.15.14-312]

\section{REFERENCES}

[1] G. Lippmann, "La Photographie Integrale, (1908)" C. R. Acad. Sci., vol. 146, pp. 446-451.

[2] F. Okano, H. Hoshino, J. Arai, and I. Yuyama, (1997) "Real-Time Pickup Method for a Three Dimensional Image Based on Integral Photography,” Appl. Opt., vol. 36, pp. 1598-1603.

[3] Y.-W. Song, B. Javidi, and F. Jin, (2005) “3D Object Scaling in Integral Imaging Display by Varying the Spatial Ray Sampling Rate,” Opt. Express, vol. 13, pp. 3242-3251.

[4] F. Okano, J. Arai, K. Mitani, and M. Okui, (2006) "Real-Time Integral Imaging Based on Extremely High Resolution Video System,” Proc. of IEEE, vol. 94, pp. 490-501.

[5] J. Arai, F. Okano, H. Hoshino, and I. Yuyama, (1998) "Gradient-index Lens Array Method Based on Real-time Integral Photography for Three-dimensional Omages,” Appl. Opt., vol. 37, pp. 2034-2045.

[6] R. Cuenca, A. Pons, G. Saavedra, M. Corral, and B. Javidi, (2006) "Optically-Corrected Elemental Images for Undistorted Integral Image Display,” Opt. Express, vol. 14, pp. 9657-9663.

[7] N. Davies, M. McCormick, and L. Yang, (1998) "Three-Dimensional Imaging Systems: A New Development," Appl. Opt., vol. 27, pp. 4520-4528.

[8] Y. A. Dudnikov, "Elimination of Image Pseudoscopy in Integral Photography, (1971) " Opt. Technology, vol. 38, pp. 140-143.

[9] S.-W. Min, J. Hong, and B. Lee, (2004) "Analysis of an Optical Depth Converter Used in a ThreeDimensional Integral Imaging System,” Appl. Opt., vol. 43, pp. 4539-4549.

[10] B. Lee, J.-H. Park and S.-W. Min, (2006) "Three-Dimensional Display and Information Processing Based on Integral Imaging," in Digital Holography and Three-Dimensional Display, (Springer, New York, USA, 2006), pp. 333-378.

[11] Ganbat Baasantseren, Jae-Hyeung Park, Ki-Chul Kwon and Nam Kim, (2009) "Viewing angle enhanced integral imaging display using two elemental image masks", Optics Express-14405, Vol.17.No.16.

[12] J.-H. Park, J. Kim, Y. Kim, and B. Lee, (2005) "Resolution-Enhanced Three-Dimension / TwoDimension Convertible Display Based on Integral Imaging," Opt. Express, vol. 13, pp. 1875-1884.

[13] J. -H. Park, J. Kim, J.- P. Bae, Y. Kim, and B. Lee, (2005) "Viewing Angle Enhancement of ThreeDimension/Two-Dimension Convertible Integral Imaging Display Using Double Collimated or Noncollimated Illumination,” Jpn. J. Appl. Phys, vol. 44, pp. 991-994. 
Authors

Md. Shariful Islam received the B.Sc. and M.Sc. degrees from the Department of Applied Physics, Electronics and Communication Engineering (Erstwhile Electronics \& Applied Physics) from Islamic University, Kushtia-7003, Bangladesh, in 1999 (Examination held 2001) and 2000 (Examination held-2003), respectively.. He joined as a lecturer in the Dept. of Information and Communication Engineering, IU, Kushtia-7003, Bangladesh, in September 2004 and currently, he is working as an Assistant Professor in the same department. Now, he is doing his PhD work on

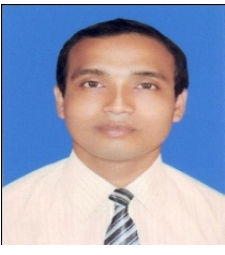
Integral Imaging Three-dimensional display (3D) system. His research interests are in the area of Integral Imaging 3D display technology. Image processing.

Md. Tariquzzaman received the B.Sc. and M.Sc. degrees from the Department of Applied Physics, Electronics and Communication Engineering (Erstwhile Electronics \& Applied Physics) from Islamic University, Kushtia-7003, Bangladesh, in 1999 (Examination held 2001) and 2000 (Examination held-2003), respectively. He received the Ph.D. degree in Engineering in 2010 from Chonnam National University, Republic of Korea. . From 2010 to 2011 he worked as a postdoctoral fellow in the School of Electronics \& Computer Engineering, Chonnam National University, and Mokpo National University Republic of Korea. He joined as a lecturer in the Dept. of Information and Communication Engineering, IU, Kushtia-7003, Bangladesh, in September 2004 and currently, he is working as an Associate Professor in the same department. His research interests are in the area of audio-visual speech processing, biometrics, computer vision and machine learning

Md. Zahidul Islam has born on December $29^{\text {th }}, 1978$ in Kushtia, Bangladesh. He has received his B.Sc. and M.Sc. degrees from the Department of Applied Physics \& Electronic Engineering, University of Rajshahi (RU), Bangladesh in 2000 and 2002 respectively. In 2003, he has joined as a Lecturer in the Department of Information and Communication Engineering, Islamic University (IU), Kushtia, Bangladesh. He has done his Ph.D research on Visual Object Tracking System from the Department of Computer Engineering under the supervision of Prof. Chil-Woo Lee in Intelligent Image Media \& Interface Lab, Chonnam National University (CNU), South Korea. In August 2011, Dr. Islam has been successfully awarded his PhD from the same department. Besides, he has done his research internship in $\mathbf{3 D}$ Vision Lab in Samsung Advanced Institute

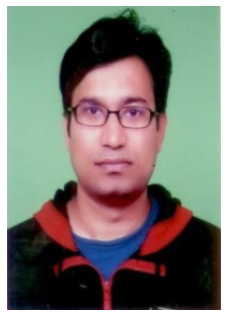
of Technology (SAIT), Suwon, South Korea. Dr. Islam has also other research interests like computer vision, 3D object, human and motion tracking and tracking articulated body, genetic algorithm etc. 